\title{
Constant Envelope Precoding by Interference Exploitation in Phase Shift Keying-Modulated Multiuser Transmission
}

\author{
Pierluigi Vito Amadori, Student Member, IEEE, and Christos Masouros, Senior Member, IEEE
}

\begin{abstract}
We introduce a new approach to constant-envelope precoding (CEP) based on an interference-driven optimization region for generic phase-shift-keying modulations in the multiuser (MU) multiple-input-multiple-output downlink. While conventional precoding approaches aim to minimize the multi-user interference (MUI) with a total sum-power constraint at the transmitter, in the proposed scheme we consider MUI as a source of additional energy to increase the signal-to-interferenceand-noise-ratio at the receiver. In our studies, we focus on two different CEP approaches: a first technique, where the power at each antenna is fixed to a specific value, and a two-step approach, where we first relax the power constraints to be lower than a defined parameter and then enforce CEP transmission. The algorithms are studied in terms of computational costs, with a detailed comparison between the proposed approach and the classical interference suppression schemes from the literature. Moreover, we analytically derive a robust optimization region to counteract the effects of channel-state estimation errors. The presented schemes are evaluated in terms of achievable symbol error rate in a perfect and imperfect channel-state information scenario for different modulation orders. Our results show that the proposed techniques further extend the benefits of classical CEP by judiciously relaxing the optimization region.
\end{abstract}

Index Terms-Constant-envelope, multiuser, massive MIMO.

\section{INTRODUCTION}

$\mathbf{M}$ ASSIVE Multiple-Input-Multiple-Output (M-MIMO) communication systems have experienced an increasing growth of interest from the scientific community, because of the significant benefits they provide in terms of spectral efficiency when compared to classical MIMO approaches [1], [2]. The pioneering work from [1] proved that a base station (BS) equipped with high dimensional antenna arrays can achieve high throughput values by exploiting the innate high degrees of freedom offered by a large number of antennas at the transmitter. At the same time, M-MIMO systems are known to require lower values of radiated energy, thanks to the higher beamforming gains provided by large antenna arrays. Moreover, it has been proven that simple linear precoding

Manuscript received February 15, 2016; revised July 5, 2016; accepted October 26, 2016. Date of publication November 8, 2016; date of current version January 6, 2017. This work was supported in part by the Royal Academy of Engineering, U.K., and in part by the Engineering and Physical Sciences Research Council project under Grant EP/M014150/1. The associate editor coordinating the review of this paper and approving it for publication was W. Su.

The authors are with the Department of Electronic and Electrical Engineering, University College London, London, WC1E 7JE, U.K. (e-mail: uceepva@ucl.ac.uk, c.masouros@ucl.ac.uk).

Color versions of one or more of the figures in this paper are available online at http://ieeexplore.ieee.org.

Digital Object Identifier 10.1109/TWC.2016.2626279 techniques, such as matched filtering and zero forcing [3], [4], are asymptotically optimal [5] for massive systems, because of the favorable propagation effects that rise for infinitely large arrays. In addition, recent works [6]-[9] have shown that transmit mutual coupling at the base station can be exploited with the aim to further increase the dimensions of antenna arrays in fixed physical spaces.

When considering linear precoding techniques, it is common in the literature [10], [11] to apply sum-power constraints at the transmitter side, where the average or instantaneous total transmitted power is constrained to a specific value. While a sum-power constraint approach is easier to model and study, it is important to consider that, in a realistic scenario, each antenna of the base station is typically connected to its own power amplifier (PA), which has to meet specific power constraints. This is particularly relevant in M-MIMO, because the benefits of using a large number of antennas at the transmitter side are followed by heavy burdens in terms of hardware costs and power consumption, which strongly affect its feasibility for future communication systems. In fact, the use of very large arrays (VLA) leads to an equally large number of radio-frequency (RF) chains, where the role of PAs is particularly critical, as inefficient PAs are accountable for $\sim 40-50 \%$ of the total power consumption [12].

Toward this end, the employment of non-linear RF components in conjunction with low peak-to-average powerratio (PAPR) precoding techniques [13] can positively affect the power efficiency of M-MIMO [14]-[16]. More specifically, [14] presents a transmission scheme for orthogonal frequency-division multiplexing (OFDM) modulations based on low PAPR precoding, while [15] and [16] propose a constant envelope precoding technique where the transmitted signal amplitude corresponding to each antenna is constant and independent from the channel realization, i.e., leading to a unitary PAPR and therefore facilitating low cost PAs. In [15] the precoding technique is designed by minimizing the error norm function of the received signal for a single user scenario, while in [16] the transmitted symbols vector is designed for multiuser MIMO with the aim to reduce the interference caused by other users. CEP was further analyzed in [17], where the precoding design for frequency-selective MIMO channels is presented. Still, the performances of CEP with interference reduction are strongly affected by the number of iterations used and by the array size at the transmitter side [16]. In addition, the study in [18] investigated the effects of phase constraints at the transmitter, since additional restrictions to the change in transmitted phases at different 
symbol times can increase the power efficiency of the system. Finally, the authors in [19] further improved the performances of interference reduction CEP, by employing cross-entropy optimization instead of gradient descent based algorithms.

While the above approaches focus on interference minimization, previous works on linear precoding [20]-[22] showed that interference minimization does not necessarily lead to the best performances in a communication system. In fact, since interference is data dependent, the transmitter is able to predict the MUI at the receiver and can use this knowledge to influence it and benefit from it. Early works in [23] and [24] focused on reducing the the negative effects of interference while preserving its positive components, defined according to the correlation between the substreams of a MIMO PSK-modulated transmission. Further results in [25] instead, showed that the transmitted signal can be precoded in order to rotate the destructive component of interference into constructive or beneficial interference. Therefore, future research is focusing onto identifying new optimization metrics that exploit channel-state information (CSI) and data knowledge at the transmitter side to maximize the signal-to-interference-andnoise-ratio (SINR) of each user by capitalizing on the power contained within multi-user interference. More specifically, recent works [26]-[30] on PSK modulated signals have introduced different metrics that prove how the known interference can be effectively used as a source of green signal power for downlink transmissions with high-order PSK modulations. Finally, [31] has investigated the applicability of symbol-level precoding based on relaxed receive constellations to increase the security of multiuser MIMO communication systems by means of Directional Modulation [32].

In this paper, we present two novel CEP techniques which exploit concepts of constructive interference for PSK-modulated signals. In the proposed techniques, we relax the conditions over interference, allowing the transmitter to use the interfering signal as a green source of power to increase the signal-to-interference-and-noise-ratio at the receiver side. It is important to highlight that the proposed schemes are particularly suitable for high-interference and low-SNR scenarios, where low-order modulations such as BPSK and 4-PSK are often preferred to ensure reliable communications [33]. Nevertheless, the benefits of constructive interference could also be extended to Quadrature Amplitude Modulation (QAM) signals, e.g., over the outer constellation points of a 16-QAM or to the whole constellation by means of adaptive decision thresholds [22]. More specifically, [34] showed the benefits of applying concepts of constructive interference over 16-QAM modulations in comparison with standard techniques, by effectively differentiating between predictable and unpredictable interference at the BS and allowing the interference to constructively superimpose with the desired signal. In a similar manner, [35] introduced constraints and metrics for symbol-level precoding based on constructive interference for QAM and asymmetric phase-shift keying (APSK) modulations, showing how such approach is able to greatly enhance power efficiency at the transmitter side. By employing similar methodologies, it is intuitive that our approach can also be extended to provide benefits for QAM constellations.
Here we list the contributions of the paper:

- We analytically describe and define a new optimization region for constant envelope precoding, based on the concepts of constructive interference.

- We introduce two different CEP approaches, when both equality and inequality power constraints are considered.

- We study the computational costs of the proposed techniques in comparison with the classical CEP approach in the literature.

- We introduce a CSI-robust precoding scheme based on a relaxation of the interference optimization region.

- We evaluate the performances of the proposed schemes for different PSK modulation orders and in scenarios where the transmitter holds perfect and imperfect CSI.

The rest of the paper is organized as follows: Section II introduces the system model used throughout this work and describes the classical CEP approach from the literature, Section III describes the proposed interference-based optimization region, while Section IV is dedicated to a thorough description of the proposed techniques. In Section V the computational complexity of the proposed scheme is analyzed and compared with the previous approach, based on interference mitigation. In Section VI a robust optimization region is analytically derived as a function of the CSI error upperbound and Section VII shows the performance achieved by the proposed techniques in different scenarios. Finally, in Section VIII the main contributions of the paper are summarized.

Notation: Upper case boldfaced letters are used for matrices (i.e., $\mathbf{X}$ ), lower case boldfaced letters denote vectors (i.e., $\mathbf{x}$ ), subindices in vectors are used to identify rows of a matrix (i.e., $\mathbf{x}_{m}$ is the $m$-th row of $\left.\mathbf{X}\right), \operatorname{tr}[\cdot]$ represents the trace of the argument and superscripts $(\cdot)^{H}$ and $(\cdot)^{*}$ stand for Hermitian transpose and complex conjugate, respectively. Operators $\Im(\cdot)$ and $\Re(\cdot)$ respectively represent the imaginary and real part of the argument.

\section{SYSTEM MODEL}

Consider a downlink multi-user scenario where the BS employs an $N$ dimensional antenna array to communicate with a population of $M$ single-antenna users. The received signal y is a $\mathbb{C}^{M \times 1}$ vector that collects the $M$ user received signals $y_{m}$, and is analytically defined as:

$$
\mathbf{y}=\mathbf{H x}+\mathbf{w},
$$

where $\mathbf{H}$ is the $\mathbb{C}^{M \times N}$ channel matrix, $\mathbf{x}$ represents the $\mathbb{C}^{N \times 1}$ vector of transmitted symbols and $\mathbf{w}$ is the $\mathbb{C}^{M \times 1}$ zero mean additive white Gaussian noise vector, i.e., $\mathbf{w} \sim \mathcal{C} \mathcal{N}\left(0, N_{0}\right)$ with $N_{0}$ being the noise variance. Complex channel gains $h_{m, n}$ in M-MIMO are modeled to include both the complex small scale fading $g_{m, n}$ between the $n$-th antenna and the $m$-th user and the real large scale fading coefficient $\beta_{m}$ experienced by the $m$-th user [1], leading to the following analytical definition

$$
h_{m, n}=g_{m, n} \sqrt{\beta_{m}} .
$$


In our studies, we consider a single cell scenario where channel gains are modeled by independent Rayleigh fading [36], i.e., small scale fading $g_{m, n}$ are zero mean i.i.d. Gaussian variables and large scale coefficients $\beta_{m}=1$, $\forall m \in\{1, \ldots, M\}$.

Given the total transmitted power by the antenna array $P_{t}$, we can define the $n$-th transmitted symbol from the $n$-th antenna of the BS as [16]

$$
x_{n}=\sqrt{P_{n}} e^{j \theta_{n}},
$$

where $P_{n}$ is the power transmitted from the $n$-th antenna, so that $\sum_{n=1}^{N} P_{n}=P_{t}$, and $\theta_{n}$ represents the precoding phase of the CEP signal. We can then similarly define the received signal at the $m$-th user as

$$
y_{m}=\sum_{n=1}^{N} h_{m, n} \sqrt{P_{n}} e^{j \theta_{n}}+w_{m} .
$$

For simplicity and to ease the notation, throughout the paper we assume unitary transmitted power $P_{t}=1$ and equally distributed power among the $N$ antennas at the BS, i.e., $P_{n}=1 / N, \forall n \in\{1, \ldots, N\}$, hence leading to

$$
y_{m}=\sum_{n=1}^{N} \frac{1}{\sqrt{N}} h_{m, n} e^{j \theta_{n}}+w_{m} .
$$

The first term of the received signal $y_{m}$ can be rearranged in order to explicitly discriminate between the desired signal and the interference. Analytically we have

$$
y_{m}=u_{m}+t_{m}+w_{m},
$$

where $u_{m}=d_{m} e^{j \phi_{m}}$ is the PSK desired symbol for the $m$-th user, with magnitude $d_{m}$ and phase $\phi_{m}$, and $t_{m}$ represents the interfering signal for the $m$-th user

$$
t_{m}=\left(\sum_{n=1}^{N} \frac{1}{\sqrt{N}} h_{m, n} e^{j \theta_{n}}-d_{m} e^{j \phi_{m}}\right) .
$$

Accordingly, we can identify the total MUI energy as

$$
E_{M U I}=\sum_{m=1}^{M}\left|\left(\sum_{n=1}^{N} \frac{1}{\sqrt{N}} h_{m, n} e^{j \theta_{n}}-d_{m} e^{j \phi_{m}}\right)\right|^{2} .
$$

First approaches to CEP were based on the minimization of the MUI energy [16]. In order to minimize (8), the base station proceeds in identifying the $N$ dimensional transmit phase angle vector $\boldsymbol{\theta}=\left[\theta_{1}, \ldots, \theta_{N}\right]$ that leads to the lowest MUI energy. Accordingly, the constant envelope precoding algorithm can be formulated as follows [16], [19]

$$
\begin{gathered}
\mathcal{P}_{1}: \quad \underset{\theta}{\operatorname{minimize}} \sum_{m=1}^{M}\left|\left(\sum_{n=1}^{N} \frac{1}{\sqrt{N}} h_{m, n} e^{j \theta_{n}}-d_{m} e^{j \phi_{m}}\right)\right|^{2} \\
\text { subject to }\left|\theta_{n}\right| \leq \pi, \quad \forall n \in\{1, \ldots, N\},
\end{gathered}
$$

which represents a non-convex nonlinear least squares (NLS) problem, affected by local minima. The optimization problem (9) was first solved in [16] with a gradient descent (GD) based approach, and further improved in [19] with a direct application of cross-entropy method [37].

\section{Constructive Interference OPTIMIZATION REGION}

When considering PSK-modulated signals, interference can be classified as constructive and destructive according to simple geometrical concepts, detailed in [23]-[27]. In fact, the interference signal $t_{m}$ can be considered beneficial for system performances when it leads the noise free received symbol $\tilde{r}_{m}=y_{m}-w_{m}$ further away from the decision thresholds of the desired constellation symbol $u_{m}$. A visual representation of the distinction between constructive and destructive interference is presented in Fig. 1a, where the desired symbol $u_{m}$ is considered to be the $(1 / \sqrt{2}+j 1 / \sqrt{2})$ point of the 8-PSK constellation. Here the superscripts $\{\cdot\}^{c}$ and $\{\cdot\}^{d}$ are used to differentiate between two different cases, where the received symbol falls in the constructive region (i.e., the green shaded area) or in destructive region (i.e., the red dot-pattern area), respectively. As per above, we can see that when the received symbol falls in the destructive region it resides closer to the decision thresholds, represented by the bold lines, when compared to the desired symbol. On the other hand, when $\tilde{r}_{m}$ lays in the constructive region, its distance from the decision thresholds is greater than the one which characterizes $u_{m}$.

Constructive interference conditions for generalized PSK modulated signals can be analytically expressed as [26]

$$
\left|\Im\left(\tilde{r}_{m} \cdot e^{-j \phi_{m}}\right)\right| \leq \Re\left(\tilde{r}_{m} \cdot e^{-j \phi_{m}}\right) \tan \Phi,
$$

where $\Phi$ is the central angle of the constructive interference sectors, which depends on the constellation order $L$ and can be readily computed as $\Phi=\pi / L$. Note that the condition in (10) is applied to the phase-shifted received signal $\tilde{r}_{m} \cdot e^{-j \phi_{m}}$, where $\phi_{m}$ is the phase of the symbol of interest for the $m$-th user. The phase-shift is a fundamental operation, as it isolates the received amplitude and phase shift over the desired symbol $u_{m}$ caused by the interference $t_{m}$. It is important to stress that these conditions are valid for any PSK modulation order.

The constructive interference constraint definition in (10) allows the identification of a new precoding optimization region that exploits the interfering signal power, instead of reducing it. In fact, as shown in Fig. 1a, the constructive interference regions are sectors with infinite radii whose central angle depends on the constellation order. This represents a relaxation from the classical optimization metrics based on interference minimization, as the constructive interference region is only constrained by the proximity to the decision thresholds and extends infinitely in the directions away from them. Optimization region constraints are visually represented in Fig. 1a by the dashed lines.

In our studies, constructive interference conditions are explicitly imposed over the interfering signal by substituting $t_{m}$ into $\tilde{r}_{m}$ as

$$
\left|\Im\left(t_{m} \cdot e^{-j \phi_{m}}\right)\right| \leq \Re\left(t_{m} \cdot e^{-j \phi_{m}}\right) \tan \Phi .
$$

The condition (11) is visually described in Fig. $1 \mathrm{~b}$ for the 8-PSK case, where $\bar{t}_{m}=t_{m} \cdot e^{-j \phi_{m}}$ represents the rotated interfering signal for the $m$-th user and $\bar{t}_{m}^{R}=\Re\left(\bar{t}_{m}\right)$ and $\bar{t}_{m}^{I}=\Im\left(\bar{t}_{m}\right)$ identify the shift from $u_{m}$ suffered by the received 


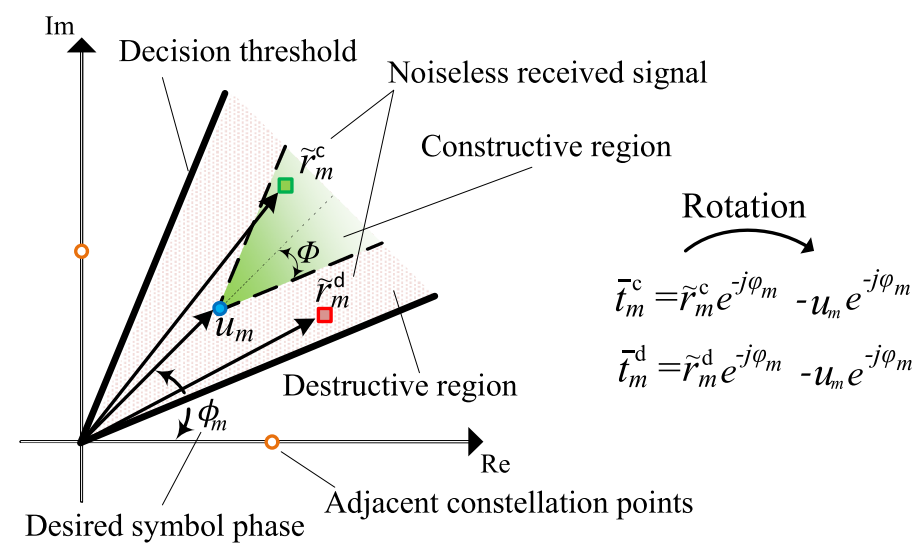

(a)

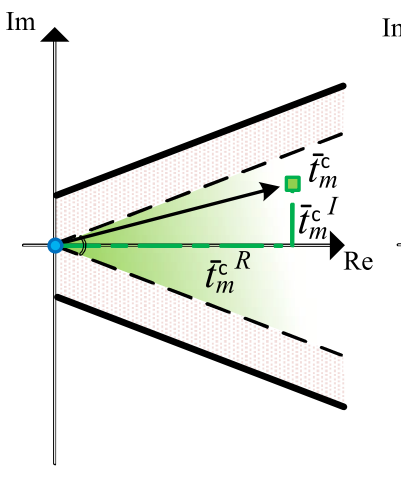

Constructive $t_{m}^{c}$

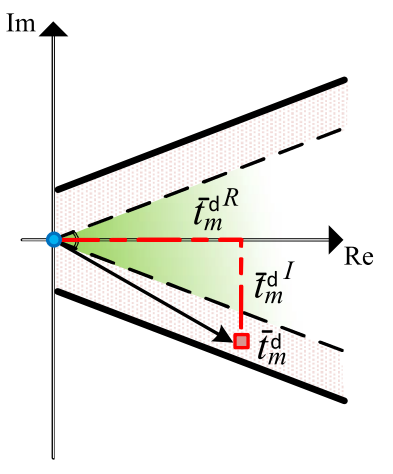

Destructive $t_{m}^{\mathrm{d}}$

(b)

Fig. 1. Optimization region for constructive interference exploitation, 8PSK example: (a) Interference regions for 8-PSK symbol, (b) Interference signal after rotation.

symbol by means of interference. More specifically, $\bar{t}_{m}^{R}$ is an analytical measure of the amplification of the received constellation point along the axis of $u_{m}$, while $\bar{t}_{m}^{I}$ represents a linear measure of the angle shift from the original constellation point with phase $\phi_{m} .{ }^{1}$ The reader is referred to [24]-[26] for more details on the definition of the constructive interference region.

\section{Constant Envelope Precoding With CONSTRUCTIVE INTERFERENCE OPTIMIZATION}

Existing studies in M-MIMO systems mostly consider precoding techniques with sum-power constraints at the transmitter side. However, this is not a realistic assumption, since each transmitting antenna is typically characterized by its own amplifier and is hence affected by specific power constraints. Moreover, the use of precoding techniques where the power at each antenna is fixed also allows the employment of powerefficient amplifiers, hence reducing the total operational power consumption of the system. Since CEP provides a solution to the above challenges, here we apply the above interference exploitation concept, to improve the performance of classical CEP approaches.

Toward this end, we introduce two different CEP approaches, both based on constructive interference exploitation concepts: one with CEP equality constraints, i.e., $\left|x_{n}\right|=$ $p, \forall n \in\{1, \ldots, N\}$, and a two-stage approach where the constraints are initially relaxed to inequality conditions, i.e., $\left|x_{n}\right| \leq p, \forall n \in\{1, \ldots, N\}$, to be successively reapplied by means of normalization in order to perform CEP.

Following the concepts of constructive interference in (11), it is possible to define a new optimization metric that maximizes the interference power, while imposing constraints over the phase of $t_{m}$. Thanks to simple analytical operations, we can rearrange (11) as

$$
\Re\left(t_{m} \cdot e^{-j \phi_{m}}\right) \tan \Phi-\left|\Im\left(t_{m} \cdot e^{-j \phi_{m}}\right)\right| \geq 0 .
$$

\footnotetext{
${ }^{1}$ It is important to stress that $\bar{t}_{m}^{R}$ and $\bar{t}_{m}^{I}$ can grow infinitely, as long they respect the condition in (10).
}

The difference on the left side of the inequation can be used as an indicator of how constructive or destructive the interfering signal $t_{m}$ is. In fact, if (12) is negative, the interfering signal lies in the destructive region of interference, while if (12) is positive it implies that the interfering signal is constructive. In addition, since the real part of (11) represents the power of the interfering signal, we can infer that higher and positive values of (12) lead to stronger forms of constructive interference. Accordingly, we define the optimization problem $\mathcal{P}_{2}$ as follows:

$\mathcal{P}_{2}: \quad \underset{\boldsymbol{\theta}}{\operatorname{maximize}} \min _{m}\left\{\Re\left(t_{m} e^{-j \phi_{m}}\right) \tan \Phi-\left|\Im\left(t_{m} e^{-j \phi_{m}}\right)\right|\right\}$ subject to $\left|\theta_{n}\right| \leq \pi, \quad \forall n \in\{1, \ldots, N\}$,

where $m \in\{1, \ldots, M\}$ and the operator $\min _{m}\{\cdot\}$ represents the minimum value of the argument among each of the $M$ values. In $\mathcal{P}_{2}$ we maximize the minimum value of the constructive interference metric. With this approach, when the minimum value of the metric is positive, we can automatically infer that the constructive interference condition is verified and maximized for all the $M$ users. In cases where the solution to $\mathcal{P}_{2}$ leads to negative values of the minimum, instead, it implies that the precoding phases minimize the destructive interference as its least constructive component is maximized, as visually described for the 8-PSK case in Fig. 1b. The formulation in $\mathcal{P}_{2}$ is clearly non-convex, however it can be efficiently solved via the cross-entropy method (CEM).

\section{A. A CEM Solver for Constructive Interference Optimization}

The cross-entropy method can be described as an adaptive algorithm that aims to the identification of rare events by means of variance reduction. The algorithm is characterized by an iterative approach [37], where each iteration presents two main steps:

- Generation of random samples based on a specific distribution $f(\boldsymbol{\theta}, \mathbf{u})$.

- Update distribution parameters $\mathbf{u} \in \mathbb{R}$, according to the computed values of a chosen cost function, in order to 
improve the random samples generation in the following iterations.

The use of cross-entropy method to perform combinatorial optimization can be described as follows. Consider the maximization problem described in $\mathcal{P}_{2}$, we can define the global optimum $\gamma^{*}$ as

$$
\begin{aligned}
\gamma^{*} & =\min _{m}\left\{\Re\left(\bar{t}_{m}^{*}\right) \tan \Phi-\left|\Im\left(\bar{t}_{m}^{*}\right)\right|\right\} \\
& =\max _{\boldsymbol{\theta} \in \Theta}\left[\min _{m}\left\{\Re\left(\bar{t}_{m}\right) \tan \Phi-\left|\Im\left(\bar{t}_{m}\right)\right|\right\}\right],
\end{aligned}
$$

where $\bar{t}_{m}^{*}$ represents the $m$-th element of the normalized interfering signal, analytically expressed as

$$
\bar{t}_{m}^{*}=\left(\sum_{n=1}^{N} \frac{1}{\sqrt{N}} h_{m, n} e^{j \theta_{n}^{*}}-d_{m} e^{j \phi_{m}}\right) e^{-j \phi_{m}},
$$

with $\theta_{n}^{*}$ being the $n$-th element of the optimal solution $\boldsymbol{\theta}^{*}$ to the optimization problem. The application of CEM to optimization problems is based on the association of the maximization problem with the probability estimation of a rare event. Given a performance threshold $\gamma$, we can evaluate the probability of the rare event $\min _{m}\left\{\Re\left(\bar{t}_{m}\right) \tan \Phi-\left|\Im\left(\bar{t}_{m}\right)\right|\right\} \geq \gamma$ as

$$
\begin{aligned}
\mathcal{L}(\gamma) & =\mathbb{P}_{\mathbf{u}}\left(\min _{m}\left\{\Re\left(\bar{t}_{m}\right) \tan \Phi-\left|\Im\left(\bar{t}_{m}\right)\right|\right\} \geq \gamma\right) \\
& =E_{\mathbf{u}}\left\{I\left\{\min _{m}\left\{\Re\left(\bar{t}_{m}\right) \tan \Phi-\left|\Im\left(\bar{t}_{m}\right)\right|\right\} \geq \gamma\right\}\right\} \\
& =\int I\left\{\min _{m}\left\{\Re\left(\bar{t}_{m}\right) \tan \Phi-\left|\Im\left(\bar{t}_{m}\right)\right|\right\} \geq \gamma\right\} f(\boldsymbol{\theta}, \mathbf{u}) d \boldsymbol{\theta},
\end{aligned}
$$

where the operator $\mathbb{P}_{\mathbf{u}}(\cdot)$ evaluates the probability of the event in argument, the operator $E_{\mathbf{u}}\{\cdot\}$ represents the expectation of the argument with respect to the distribution $f(\boldsymbol{\theta}, \mathbf{u})$ and $I\{\cdot\}$ is boolean indicator function that returns 1 or 0 values when its argument it true or false, respectively. The estimation of $\mathcal{L}(\gamma)$ can be performed through Monte Carlo simulations, ${ }^{2}$ by drawing a set of $K$ random states $\boldsymbol{\Theta}_{1}, \ldots, \boldsymbol{\Theta}_{K}$ from $f(\boldsymbol{\theta}, \mathbf{u})$ and by computing

$$
\widehat{\mathcal{L}}(\gamma)=\frac{1}{K} \sum_{k=1}^{K} I\left\{\min _{m}\left\{\Re\left(\bar{t}_{m}^{k}\right) \tan \Phi-\left|\Im\left(\bar{t}_{m}^{k}\right)\right|\right\} \geq \gamma\right\},
$$

where $\bar{t}_{m}^{k}$ is the $m$-th element of the interfering signal for the $k$-th state $\Theta_{k}=\left[\Theta_{1}^{k}, \ldots, \Theta_{n}^{k}, \ldots, \Theta_{N}^{k}\right]$

$$
\bar{t}_{m}^{k}=\left(\sum_{n=1}^{N} \frac{1}{\sqrt{N}} h_{m, n} e^{j \Theta_{n}^{k}}-d_{m} e^{j \phi_{m}}\right) e^{-j \phi_{m}} .
$$

A direct application of (17) becomes rapidly prohibitive when the probability of the event is very small, i.e., on the order of $\sim 10^{-5}$. This can be addressed by means of importance sampling, where we estimate a different probability density function $g(\boldsymbol{\theta})$ that more frequently generates

\footnotetext{
${ }^{2}$ While analytical estimations of $\mathcal{L}(\gamma)$ can also be performed, Monte-Carlo estimation represents the standard procedure for applications of the CrossEntropy solver, as described in [37] and [38] and as performed in [19].
}

such rare events. Under importance sampling, the estimation problem becomes

$$
\begin{aligned}
& \widehat{\mathcal{L}}(\gamma) \\
& =\frac{1}{K} \sum_{k=1}^{K} I\left\{\min _{m}\left\{\Re\left(\bar{t}_{m}^{k}\right) \tan \Phi-\left|\Im\left(\bar{t}_{m}^{k}\right)\right|\right\} \geq \gamma\right\} \frac{f\left(\boldsymbol{\Theta}_{k}, \mathbf{u}\right)}{g\left(\boldsymbol{\Theta}_{k}\right)},
\end{aligned}
$$

where $g\left(\boldsymbol{\Theta}_{k}\right)$ represents the importance sampling distribution and $\frac{f\left(\boldsymbol{\Theta}_{k}, \mathbf{u}\right)}{g\left(\boldsymbol{\Theta}_{k}\right)}$ is defined as the likelihood ratio (LR) estimator.

The importance sampling function is commonly chosen as a probability density function from the same family of $f(\boldsymbol{\theta}, \mathbf{u})$, as

$$
g(\boldsymbol{\theta})=f(\boldsymbol{\theta}, \mathbf{v}),
$$

where $\mathbf{v} \in \mathbb{R}$ is the tilting parameters vector and is obtained by computing the function with the minimum Kullback-Leiber distance from the ideal solution $g^{*}(\boldsymbol{\theta})=\frac{I\{\mathcal{S}(\boldsymbol{\theta}) \geq \gamma\} f(\boldsymbol{\theta}, \mathbf{u})}{\mathcal{L}(\gamma)}$, where $\mathcal{S}(\boldsymbol{\theta})$ is a real valued function of the optimization parameter $\boldsymbol{\theta}$. The Kullback-Leiber distance or cross-entropy between two densities $s(\mathbf{x})$ and $t(\mathbf{x})$ is analytically defined as

$$
\mathcal{D}(s, t)=\int s(\mathbf{x}) \ln s(\mathbf{x}) d \mathbf{x}-\int s(\mathbf{x}) \ln t(\mathbf{x}) d \mathbf{x},
$$

and its minimization can be achieved through the maximization of the second term in the equation. The tilting parameters $\mathbf{v}$ deriving from the minimization of the Kullback-Leiber distance between $g^{*}(\boldsymbol{\theta})$ and $f(\boldsymbol{\theta}, \mathbf{u})$ can be obtained as

$$
\mathbf{v}^{*}=\arg \max _{\mathbf{v}} \int \frac{I\{\mathcal{S}(\boldsymbol{\theta}) \geq \gamma\} f(\boldsymbol{\theta}, \mathbf{u})}{\mathcal{L}(\gamma)} \ln f(\boldsymbol{\theta}, \mathbf{v}) d \boldsymbol{\theta},
$$

which, for the proposed optimization problem, is equivalent to the maximization [37] :

$$
\begin{aligned}
\mathbf{v}^{*}=\arg \max _{\mathbf{v}} E_{\mathbf{u}}\left\{I\left\{\min _{m}\left\{\Re\left(\bar{t}_{m}\right) \tan \Phi-\left|\Im\left(\bar{t}_{m}\right)\right|\right\} \geq \gamma\right\}\right. \\
\times \ln f(\boldsymbol{\Theta}, \mathbf{v})\} .
\end{aligned}
$$

A solution to (23) can be numerically estimated as

$$
\begin{aligned}
\widehat{\mathbf{v}}^{*}= & \frac{1}{K} \sum_{k=1}^{K} I\left\{\min _{m}\left\{\Re\left(\bar{t}_{m}^{k}\right) \tan \Phi-\left|\Im\left(\bar{t}_{m}^{k}\right)\right|\right\} \geq \gamma\right\} \\
& \times \ln f\left(\boldsymbol{\Theta}_{k}, \mathbf{v}\right) .
\end{aligned}
$$

In our study we consider $f(\boldsymbol{\theta}, \mathbf{v})$ to be a Gaussian distribution, i.e. $f(\boldsymbol{\theta}, \mathbf{v})=f(\boldsymbol{\theta},[\mu, \sigma]),{ }^{3}$ which allows to analytically estimate (23) as

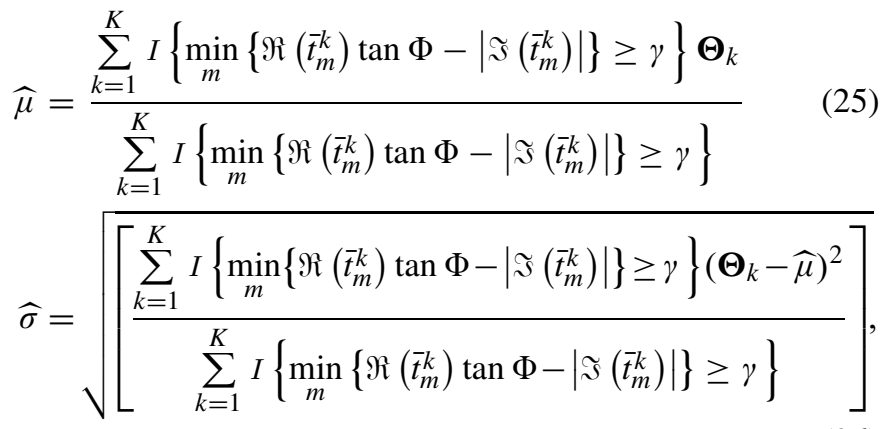

\footnotetext{
${ }^{3}$ This assumption is not uncommon for continuous optimization problems [19] and leads to efficient solutions.
} 


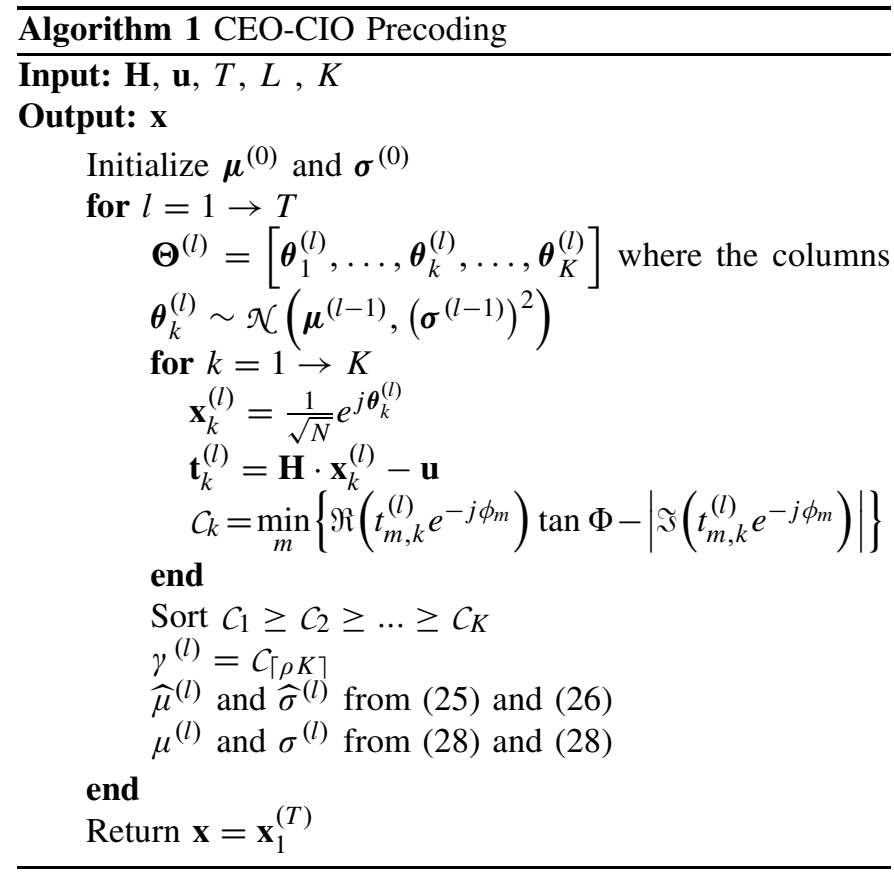

where $\widehat{\mu}$ and $\widehat{\sigma}$ respectively represent mean and standard deviation of the importance sampling distribution, i.e., $\widehat{\mathbf{v}}^{*}=$ $[\widehat{\mu}, \widehat{\sigma}]$. As previously mentioned, CEM is based on an iterative approach and requires the tilting parameters to be updated at each iteration. However, a direct update from (24) is often undesirable, as it might rapidly converge to suboptimal solutions [37]. The occurrence of these events can be reduced by using smooth updating procedures, as follows

$$
\begin{aligned}
& \mu^{(l)}=\alpha \widehat{\mu}^{(l)}+(1-\alpha) \mu^{(l-1)} \\
& \sigma^{(l)}=\alpha \widehat{\sigma}^{(l)}+(1-\alpha) \sigma^{(l-1)},
\end{aligned}
$$

where the superscript $(\cdot)^{(l)}$ represents the $l$-th iteration of the value in argument.

An analytical description of the constructive interference optimization precoding based on cross-entropy optimization (CEO-CIO) technique is presented in Algorithm 1. Here, $T$ represents the number of iterations, $K$ identifies the random sample size and $\rho$ is direct proportionality coefficient used to compute the intermediate threshold $\gamma^{(l)}$. More specifically, the intermediate threshold $\gamma^{(l)}$ is identified by the cost function evaluation $C_{k}$ whose index is the smallest integer to be greater or equal to $\rho K$ and is evaluated in Algorithm 1 as $\mathcal{C}_{\lceil\rho K\rceil}$.

The application of the proposed algorithm leads to received symbols $\tilde{\mathbf{r}}$ which prevalently reside in the constructive interference region. To illustrate this effect, Fig. 2 shows the received constellation of CEP precoded signals for the example of 8-PSK constellation in a noise free transmission over 100 different channel realizations, in a scenario where the BS is equipped with $N=100$ antennas and communicates with $M=20$ single-antenna users.

\section{B. Two-Step Convex CEP}

In addition to the previous approach, we propose an additional technique for constant envelope transmissions where

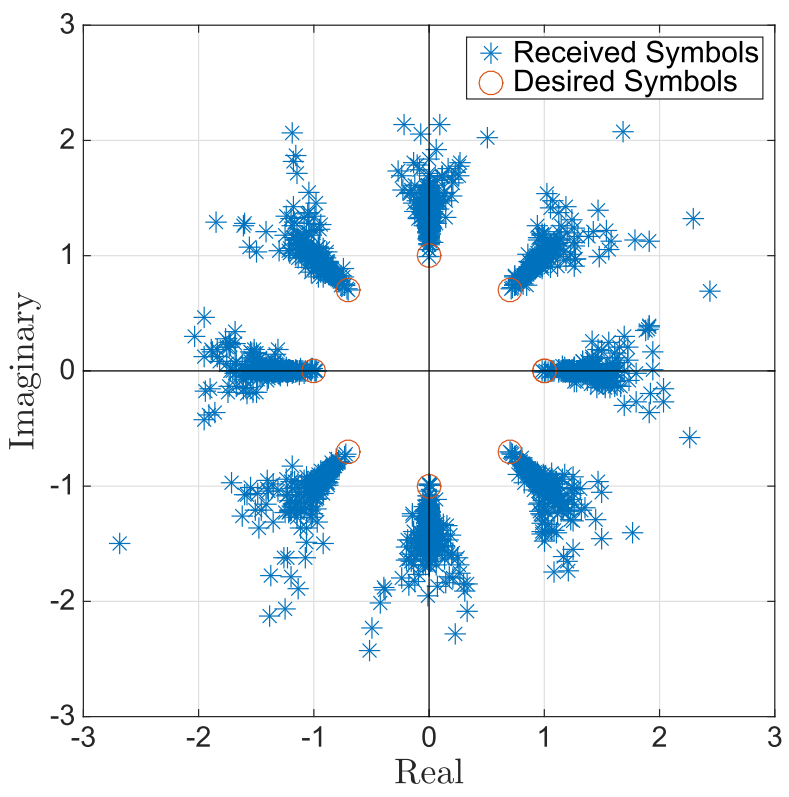

Fig. 2. Received symbols in a noiseless scenario with $N=100$ antennas for $M=20$ users when using 8-PSK.

the power constraints are initially relaxed into inequality, allowing to use standard convex optimization techniques, and subsequently enforced to equality via normalization at a later stage (i.e., by dividing the antenna outputs that do not respect power constraints by their absolute value). In order to relax the conditions in $\mathcal{P}_{2}$, we reformulate the optimization problem in its equivalent form where the cost function is dependent on the transmitted signal $\mathbf{x}$ :

$$
\begin{array}{r}
\mathcal{P}_{3}: \quad \underset{x}{\operatorname{maximize}} \min _{m}\left\{\Re\left(\bar{t}_{m}\right) \tan \Phi-\left|\Im\left(\bar{t}_{m}\right)\right|\right\} \\
\text { subject to }\left|x_{n}\right|=1 / \sqrt{N}, \quad \forall n \in\{1, \ldots, N\}, \\
\bar{t}_{m}=\left(\sum_{n=1}^{N} h_{m, n} x_{n}-u_{m}\right) e^{-j \phi_{m}} .
\end{array}
$$

Similarly to the optimization in $\mathcal{P}_{2}$, the above problem is non-convex, because of the equality constraint over a convex set. In order to tackle this, we can convexify the problem by imposing relaxed conditions to the transmitted signal $x_{n} \in \mathbb{C}, \forall n \in\{1, \ldots, N\}$ and its absolute value $\left|x_{n}\right| \leq$ $1 / \sqrt{N}, \forall n \in\{1, \ldots, N\}$. Thanks to this, we can reformulate the optimization problem $\mathcal{P}_{3}$ into its relaxation $\mathcal{P}_{3}^{\prime}$ as

$$
\begin{array}{r}
\mathcal{P}_{3}^{\prime}: \quad \underset{\boldsymbol{x}^{\prime}}{\operatorname{maximize}} \min _{m}\left\{\Re\left(\bar{t}_{m}\right) \tan \Phi-\left|\mathfrak{\Im}\left(\bar{t}_{m}\right)\right|\right\} \\
\text { subject to }\left|x_{n}^{\prime}\right| \leq 1 / \sqrt{N}, \quad \forall n \in\{1, \ldots, N\}, \\
\bar{t}_{m}=\left(\sum_{n=1}^{N} h_{m, n} x_{n}^{\prime}-u_{m}\right) e^{-j \phi_{m}},
\end{array}
$$

where the superscript $\{\cdot\}^{\prime}$ is used to identify the solution achieved through relaxation. Differently from $\mathcal{P}_{3}$, the newly formulated problem is a standard second-order cone 


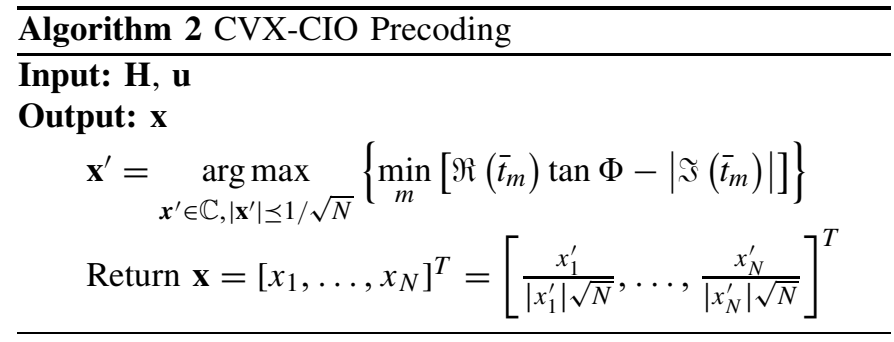

program (SOCP) ${ }^{4}$ and can be effectively solved by means of standard convex optimization techniques [26]. Since the constraints over the amplitude of the precoded signal $\left|x_{n}^{\prime}\right| \leq$ $1 / \sqrt{N}, \forall n \in\{1, \ldots, N\}$ cannot guarantee a strict constant envelope condition, in order to achieve a full CEP transmission for all the antennas at the BS we need to force the equality constrained before transmission. More specifically, in the second and final stage of the algorithm, we can proceed by normalizing the elements where $\left|x_{n}^{\prime}\right| \neq 1 / \sqrt{N}, \forall n \in$ $\{1, \ldots, N\}$ as follows

$$
x_{n}= \begin{cases}x_{n}^{\prime} /\left(\sqrt{N}\left|x_{n}^{\prime}\right|\right) & \forall n \text { where }\left|x_{n}^{\prime}\right| \neq 1 / \sqrt{N} \\ x_{n}^{\prime} & \forall n \text { where }\left|x_{n}^{\prime}\right|=1 / \sqrt{N} .\end{cases}
$$

The precoding scheme, which we refer to as Convex Constructive Interference Optimization (CVX-CIO), is analytically described in Algorithm 2, where $\mathbf{x}^{\prime} \preceq 1 / \sqrt{N}$ is used to represent that $x_{n}^{\prime} \leq 1 / \sqrt{N}, \forall n \in\{1, \ldots, N\}$.

\section{Computational Complexity}

In this section, we compute and analyze the complexity of the proposed CEO-CIO in comparison with the CEO approach to interference reduction (CEO-IR) precoding from [19] in terms of floating-point operations (flops), following the operational costs listed in the literature [39]. More specifically, we consider addition, subtraction and multiplication between two floating-point numbers as a flop. Since both approaches are characterized by the same number of iterations $T$, we focus our analysis on the computational burdens of the two different cost functions.

For our study, we consider a simple time-division duplexing (TDD) scenario [40] where coherence time $T_{\text {cohe }}$ indicates the maximum number of data symbols that can be transmitted within a channel realization, i.e., when the elements of the channel matrix $\mathbf{H}$ can be considered constants. The TDD assumption is not uncommon in M-MIMO literature, as it allows to exploit the reciprocity of the channel, enabling the CSI acquisition for downlink via uplink pilots. This property is fundamental in M-MIMO systems, as the time required by CSI acquisition $T_{C S I}$ becomes proportional to the number of users $M$ instead of the number of antennas $N$. In our analysis, we consider a simple TDD case where $T_{C S I}=\mu M$, with $\mu \geq 1$ being the number of pilot slots.

\footnotetext{
${ }^{4}$ More specifically, the problem can be cast as a standard SOCP [39], for its objective function is concave [26] as it can be decomposed into the combination of a linear function $\Re\left(t_{m} e^{-j \phi_{m}}\right)$ and a concave function $-\left|\Im\left(t_{m} e^{-j \phi_{m}}\right)\right|$. In fact, in [26] it was shown that the extraction of the imaginary and real of a linear function preserves its linearity.
}

Finally, we consider a symmetrical transmission case where the time for data transmission $T_{\text {data }}=T_{\text {cohe }}-T_{C S I}$ is divided between downlink and uplink transmissions according to a parameter $0 \leq \epsilon_{D L} \leq 1$. The parameter $\epsilon_{D L}$ explicitly represents the portion of $T_{\text {data }}$ devoted to downlink symbol transmission. Analytically, we have

$$
T_{D L}=\epsilon_{D L}\left(T_{\text {cohe }}-T_{C S I}\right)=\epsilon_{D L}\left(T_{\text {cohe }}-\mu M\right) .
$$

\section{A. CEO-CIO Costs}

As previously mentioned, main costs of the proposed CEO$\mathrm{CIO}$ algorithm reside in the need to compute the cost function for each of the randomly generated samples. We can synthesize the computation of the cost function in the following main operations:

- Computation of the received vector in a noise free scenario $\tilde{\mathbf{r}}=\mathbf{H x}$,

- Identification of the interfering signal vector $\mathbf{t}=\tilde{\mathbf{r}}-\mathbf{u}$,

- Projection of the interfering signal $\overline{\mathbf{t}}=\mathbf{t} \circ \mathbf{u}^{*}$,

- Identification of $\min \{\Re(\overline{\mathbf{t}}) \tan \Phi-|\Im(\overline{\mathbf{t}})|\}$, where $\circ$ represents the Hadamard product.

From the literature [39], we know the costs of each of the aforementioned operations: the multiplication between a $M \times N$ matrix and an $N \times 1$ vector requires $M(2 N-1)$ flops, while the computation of the interfering signal and its rotation can be performed with $M$ flops each, since they can be achieved by $M$ subtractions and multiplications, respectively. Finally, we can compute the costs of the identification of the minimum as a search through an $M$-sized vector, hence leading to $M$ flops. It follows that the proposed approach is characterized by a total flop count of $M(2 N-1)+4 M$ flops, which includes the cost of the separation between the real and imaginary part of the rotated interfering signal. Computational costs for the derivation and transmission of a CEO-CIO signal are listed in Table I.

\section{B. CEO-IR Costs}

The application of the conventional CEO-IR follows a similar pattern to CEO-CIO, due to the fact that they both require the computation of the interfering signal for all the randomly generated samples. More specifically, the computational costs of CEO-IR can be highlighted in the following operations:

- Computation of the received vector in a noise free scenario $\tilde{\mathbf{r}}=\mathbf{H x}$,

- Identification of the interfering signal vector $\mathbf{t}=\tilde{\mathbf{r}}-\mathbf{u}$,

- Computation of the interference energy $\sum_{1}^{M}\left|t_{m}\right|^{2}$.

Following a similar approach to the previous section, we identify the multiplication costs in $M(2 N-1)$ flops and the computation of the interfering signal as $M$ flops. Since the interfering energy can be computed as the inner product of two $M$-sized vectors, i.e., by a cost of $2 M-1$ flops, the total cost of the CEO-IR algorithm is $M(2 N-1)+3 M-1$ flops.

As we can see, the computational costs of the proposed technique CEO-CIO are comparable to the ones of the CEO-IR approach from the literature, as the flop count difference is almost negligible. The total costs of the application 
TABLE I

COMPUTATIONAL Costs In FLOPS

\begin{tabular}{|l|c|}
\hline \multicolumn{2}{|c|}{ CEO-CIO } \\
\hline$\tilde{\mathbf{r}}=\mathbf{H x}$ & $T \cdot K \cdot M(2 N-1)$ \\
\hline $\mathbf{t}=\tilde{\mathbf{r}}-\mathbf{u}$ & $T \cdot K \cdot M$ \\
\hline$\overline{\mathbf{t}}=\mathbf{t} \circ \mathbf{u}^{*}$ & $T \cdot K \cdot M$ \\
\hline $\min \{\Re(\overline{\mathbf{t}}) \tan \Phi-|\Im(\overline{\mathbf{t}})|\}$ & $T \cdot K \cdot 2 M$ \\
\hline Total & $T_{D L} \cdot T \cdot K[M(2 N-1)+4 M]$ \\
\hline \multicolumn{2}{|c|}{ CEO-IR } \\
\hline$\tilde{\mathbf{r}}=\mathbf{H x}$ & $T \cdot K \cdot M(2 N-1)$ \\
\hline $\mathbf{t}=\tilde{\mathbf{r}}-\mathbf{u}$ & $T \cdot K \cdot M$ \\
\hline $\mathbf{t}^{H} \mathbf{t}$ & $T \cdot K \cdot 2 M-1$ \\
\hline Total & $T_{D L} \cdot T \cdot K[M(2 N-1)+3 M-1]$ \\
\hline
\end{tabular}

of the precoding techniques in a coherence time are listed in Table I, which includes the effects deriving by both the number of iterations $T$ and the sample size $K$.

\section{CSI-Robust Constant Envelope Precoding}

In the previous sections we assumed the transmitter to possess a perfect knowledge over the channel, allowing the definition of the constructive and destructive regions of interference in absence of uncertainty. When the CSI acquisition is imperfect, however, the received signal region extends according to the CSI error. We consider the BS to be aware of an estimated channel matrix, defined analytically as follows [26]

$$
\widehat{\mathbf{H}}=\mathbf{H}+\mathbf{S},
$$

where the error matrix $\mathbf{S}$ represents the CSI uncertainty at the BS, statistically independent from $\mathbf{H}$, and characterized as a constrained spherical error, i.e., each element $s_{m, n}$ : $\left\{\left|s_{m, n}\right|^{2} \leq \delta_{m, n}^{2}\right\}$ [26]. Following [26], we consider a scenario where the base station is aware of the error bounds $\delta_{m, n}^{2}$ but has no knowledge over the error matrix $\mathbf{S}$. Differently from classical robust precoding approaches from the literature [26], [41], [42], where the transmitted power is increased in order to overcome the effects of CSI estimation errors, we propose a worst-case approach where the optimization region is redefined according to the CSI uncertainty, while preserving CEP constraints. The estimated interfering signal for the $m$-th user, in case of imperfect CSI, can be defined as follows

$$
\begin{aligned}
\widehat{t}_{m} & =\left(\sum_{n=1}^{N} \frac{1}{\sqrt{N}} \widehat{h}_{m, n} e^{j \theta_{n}}-d_{m} e^{j \phi_{m}}\right) \\
& =\left[\sum_{n=1}^{N} \frac{1}{\sqrt{N}}\left(h_{m, n}+s_{m, n}\right) e^{j \theta_{n}}-d_{m} e^{j \phi_{m}}\right] \\
& =\left(\sum_{n=1}^{N} \frac{1}{\sqrt{N}} h_{m, n} e^{j \theta_{n}}-d_{m} e^{j \phi_{m}}\right)+\sum_{n=1}^{N} \frac{s_{m, n}}{\sqrt{N}} e^{j \theta_{n}},
\end{aligned}
$$

where $s_{m, n}$ represents the $n$-th element of the $m$-th row of the CSI uncertainty matrix $\mathbf{S}$. As we can see in the last step of (34), the estimated interference signal $\widehat{t}_{m}$ is characterized by two different components: the actual interference signal $t_{m}$, i.e., when considering perfect CSI, and the uncertainty

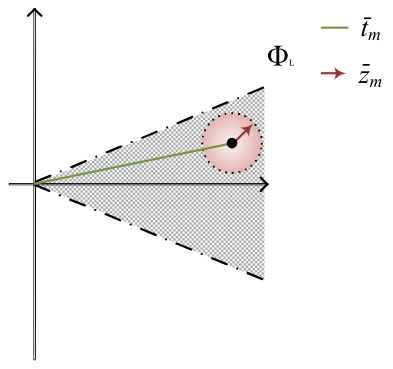

a) General case

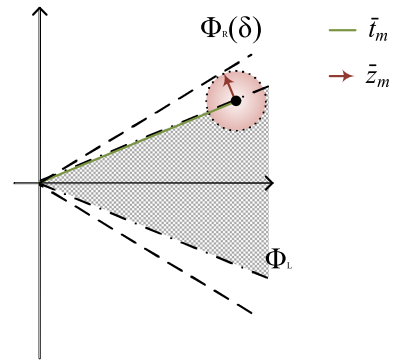

b) Worst case
Fig. 3. Imperfect CSI effects on the phase-shifted interfering signal $\bar{t}_{m}$.

error signal $z_{m}=\sum_{n=1}^{N} s_{m, n} e^{j \theta_{n}}$. It follows that the estimated interfering signal can be defined as the sum of the two terms

$$
\widehat{t}_{m}=t_{m}+z_{m} .
$$

In (11), the interfering signal is rotated according to the desired symbol, with the aim to have a region definition that is independent from the specific phase of the symbol of interest $u_{m}$. In a similar manner, we can define the rotated interfering signal for the $m$-th user in presence of CSI errors $\widehat{\bar{t}}_{m}$ as

$$
\widehat{\bar{t}}_{m}=\widehat{t}_{m} \cdot e^{-j \phi_{m}}=\bar{t}_{m}+\bar{z}_{m} .
$$

The second term in (36) can be described as the shift from the ideal interfering signal $\bar{t}_{m}$ caused by the CSI errors and can be represented as a circular constrained region of uncertainty, as visually presented in Fig. 3a. Accordingly, we can identify the worst-case scenario in the event where the actual interfering signal $\bar{t}_{m}$ is within the constructive interference region, but the uncertainty error signal $\bar{z}_{m}$ moves the estimated $\widehat{t}_{m}$ away from it, as shown in Fig. 3b. Since we assume the CSI errors to be constrained within a spherical region, it is possible to analytically derive amplitude and phase of the worst-case scenario uncertainty error signal $\bar{z}_{m}$.

Lemma 1:The amplitude of $\bar{z}_{m}$ is characterized by the following analytical upperbound

$$
\left|\bar{z}_{m}\right| \leq \frac{\sum_{n=1}^{N} \delta_{m, n}}{\sqrt{N}}
$$

Proof: Following the definition of $\bar{z}_{m}$ we have

$$
\begin{aligned}
\left|\bar{z}_{m}\right| & =\left|\sum_{n=1}^{N} \frac{1}{\sqrt{N}} s_{m, n} e^{j \theta_{n}} e^{-j \phi_{m}}\right| \\
& =\left|\sum_{n=1}^{N} \frac{1}{\sqrt{N}}\right| s_{m, n}\left|e^{j\left(\mathfrak{U}\left\{s_{m, n}\right\}+\theta_{n}-\phi_{m}\right)}\right|,
\end{aligned}
$$

where $s_{m, n}$ has been represented in order to show amplitude and phase and the operator $\mathfrak{U}\{\cdot\}$ identifies the phase extraction of the argument. The absolute value of $z_{m}$ is evaluated as the absolute value of the sum of complex values. According to the triangle inequality (i.e., given two complex numbers $a, b \in \mathbb{C}$ 
they satisfy the property $|a+b| \leq|a|+|b|)$ we have

$$
\left|\sum_{n=1}^{N} \frac{1}{\sqrt{N}}\right| s_{m, n}\left|e^{j\left(\mathfrak{U}\left\{s_{m, n}\right\}+\theta_{n}-\phi_{m}\right)}\right| \leq \sum_{n=1}^{N} \frac{1}{\sqrt{N}}\left|s_{m, n}\right| .
$$

Given the assumption of a spherical constrained error during CSI estimation, we have

$$
\sum_{n=1}^{N} \frac{1}{\sqrt{N}}\left|s_{m, n}\right| \leq \sum_{n=1}^{N} \frac{1}{\sqrt{N}} \delta_{m, n},
$$

which ends the proof.

Finally, the worst-case scenario phase of $\bar{z}_{m}$ can be readily identified as the phase that is orthogonal to the constructive interference threshold identified by $\Phi$.

The knowledge of the worst-case effects of CSI errors at the transmitter can be used to relax of the optimization region, in order to include the events that would be affected by the uncertainty error signal. Thanks to this relaxation, we can achieve a CSI errors robust precoding, without the need to increase the transmitted power.

More specifically, according to simple geometrical analysis, the phase threshold $\Phi$ is relaxed as

$$
\Phi_{R}\left(\delta_{m}\right)=\Phi_{L}+\arctan \left\{\frac{\sum_{n=1}^{N} \delta_{m, n}}{E\left\{\left|t_{m}\right|\right\} \sqrt{N}}\right\},
$$

where $\Phi_{L}=\pi / L$ identifies the threshold angle for the $L$ order PSK modulation used in transmission. Accordingly, we can define a new optimization problem, specifically designed for the imperfect CSI case

$$
\begin{array}{ll}
\mathcal{P}_{4}: & \underset{\theta}{\operatorname{maximize}} \min _{m}\left\{\Re\left(\bar{t}_{m}\right) \tan \Phi_{R}\left(\delta_{m}\right)-\left|\Im\left(\bar{t}_{m}\right)\right|\right\} \\
& \text { subject to }\left|\theta_{n}\right| \leq \pi, \forall n \in\{1, \ldots, N\} .
\end{array}
$$

Without loss of generality, in our studies we consider a case where $\delta_{m, n}=\delta, \forall m \in\{1, \ldots, M\}, \forall n \in\{1, \ldots, N\}$, which leads to a simplified definition of the robust relaxation

$$
\Phi_{R}(\delta)= \begin{cases}\Phi_{L}+\arctan \left\{\frac{\delta \sqrt{N}}{E\left\{\mid t_{m}\right\}}\right\} & \text { if } \arctan \left\{\frac{\delta \sqrt{N}}{E\left\{\left\{t_{m}\right\}\right.}\right\} \leq \frac{\pi}{L} \\ \Phi_{L-1}-\epsilon & \text { otherwise, }\end{cases}
$$

where $\epsilon$ is an arbitrarily small positive quantity, which imposes an upperbound to the growth of $\Phi_{R}$ for high values of $\delta$, and $L-1$ identifies the modulation order which is immediately lower than the one used during data transmission. The defined upperbound is particularly important, given the fact that very high values of $\delta$ could cause ambiguity with lower modulation orders, i.e., when their values lead the robust region $\Phi_{R}(\delta)$ to coincide with or exceed $\Phi_{L-1}$.

\section{RESULTS}

This section shows the performances of the proposed precoding techniques through Monte Carlo simulations over 50000 channel realizations. We consider the downlink transmission described in the previous sections, where the BS

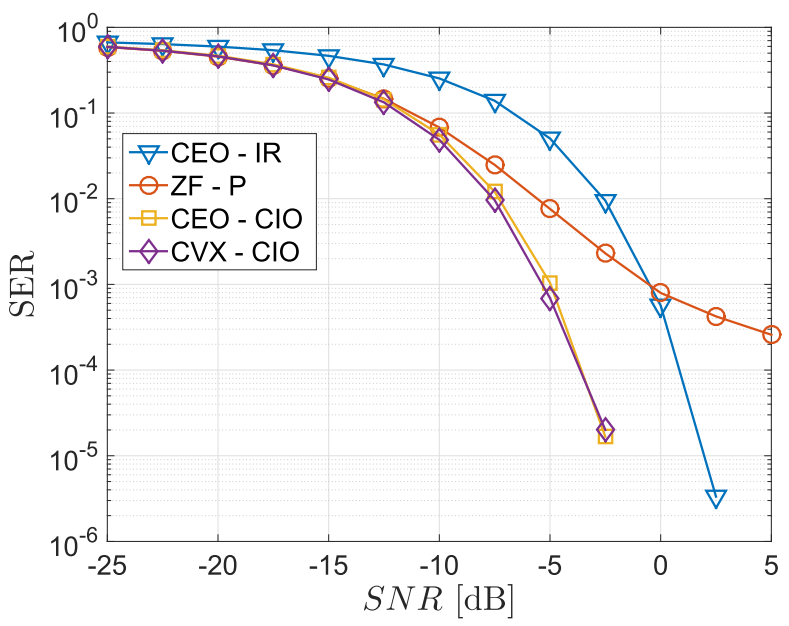

Fig. 4. 4-PSK symbol error rate when $M=12, N=64$ with perfect CSI.

employs $N=64$ antennas to communicate with a population of $M=12$ mobile users. Since the proposed technique can be applied independently from the modulation order, results are presented for both 4-PSK and 8-PSK, even though the proposed schemes can be straightforwardly applied to higher order PSK modulations. Legends are characterized by the following notation: CEO-CIO identifies constructive interference driven precoding based on CEM, CEO-IR is used to represent interference minimization CEO precoding and finally, CVX-CIO represents the two-step convex CEP approach to constructive interference optimization. Both CEO techniques are applied while considering the same parameter settings: $T=1000, \rho=0.05$ and $\alpha=0.08$ [19]. CEM solver parameter values have been studied in [38], where the used settings are recommended for achieving good performances in terms of both convergence speed and quality of the solution. In addition to CEO-IR, we compare the proposed techniques with a CEP approach to linear zero-forcing(ZF) precoding [16], ZF-P in the legends, which can be analytically defined as

$$
\mathbf{x}_{Z F-P}=\frac{e^{j \mathfrak{U}\left\{\mathbf{G}_{Z F} \mathbf{u}\right\}}}{\sqrt{N}}
$$

where $\mathbf{G}_{Z F}=\mathbf{H}^{H}\left(\mathbf{H H}^{H}\right)^{-1}$ is the ZF precoding matrix.

Figures 4 and 5 present the symbol error rate (SER) as a function of the transmitted SNR for 4-PSK and 8-PSK modulation respectively when considering a BS with $N=64$ and $M=12$ users. As we can see from Fig. 4 and Fig. 5, the proposed approaches strongly outperform the classical CEO-IR and ZF-P. This is due to the fact that CEO-CIO wisely exploits the interference signal $t_{m}, \forall m \in\{1, \ldots, M\}$ to increase the received signal power, while CEO-IR aims to a direct minimization of the interference energy. Regarding the ZF-P approach, we can see that a direct normalization of the precoded signal leads to a significant decrease in performances, due to its sub-obtimal approach. In addition, in Fig. 6 we also show the SER as a function of the transmitted SNR when a different topology is considered, where $N=32$ and $M=6$, for both the 4-PSK and 8-PSK case. Even in this different topology, the same performance trend is preserved 


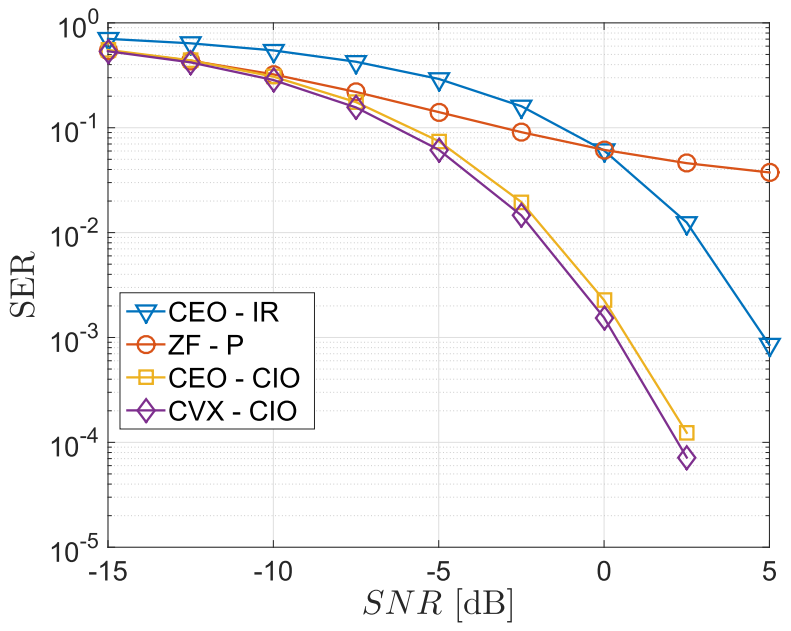

Fig. 5. 8-PSK symbol error rate when $M=12, N=64$ with perfect CSI.

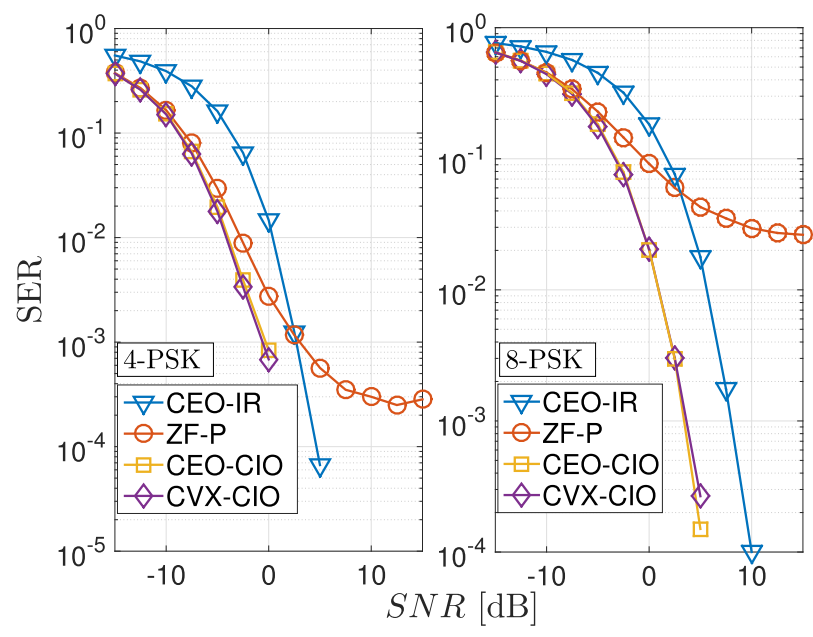

Fig. 6. Symbol error rate as a function of the transmitted SNR when $M=6$, $N=32$ with perfect CSI.

for all the described techniques, with the proposed schemes outperforming the CEP approaches from the literature. It is interesting to notice that in Fig. 6 CEO-CIO is able to achieve slightly better performances than CVX-CIO, differently from what happens in Fig.s 4-5. This is due to the fact that the second-step normalization of CVX-CIO, enforced in order to achieve a CEP transmission, has a stronger impact over the performances achievable by systems with a lower number of transmitting antennas and users, ${ }^{5}$ such as the scenario shown in Fig. 6.

In Fig. 7 we further characterize the proposed schemes when the base station possesses imperfect CSI in the scenario where $\delta_{m, n}^{2}=\delta^{2}=0.1, \forall m \in\{1, \ldots, M\}, \forall n \in\{1, \ldots, N\}$. In our studies, we set the value of $\epsilon=0.1$ and consider a simplified derivation of the relaxation $\Phi_{R}(\delta)$ where we impose

\footnotetext{
${ }^{5}$ It was empirically observed that on average both scenarios are characterized by the same number of elements where $\left|x_{n}^{\prime}\right| \neq 1 / \sqrt{N}, \forall n \in\{1, \ldots, N\}$. This means that for scenarios with a larger number of transmitting antennas, the percentage of elements where the normalization is necessary is lower, hence reducing the degradation in performances caused by the second-step normalization of CVX-CIO.
}

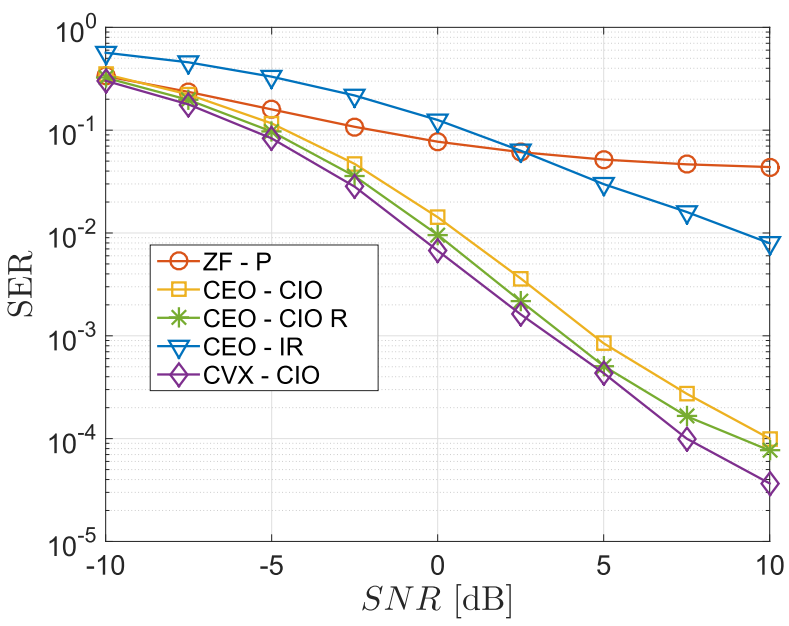

Fig. 7. Symbol error Rate for 8-PSK modulation when $M=12, N=64$ with imperfect CSI and $\delta^{2}=0.1$.

a unitary $E\left\{\left|t_{m}\right|\right\}$. While more complex derivations of $E\left\{\left|t_{m}\right|\right\}$ are expected to give a finer evaluation of $\Phi_{R}(\delta)$, it was empirically shown that such approximation has a negligible impact in the overall system performance. More specifically, Fig. 7 shows that for a system with imperfect CSI, the performances of classical CEO-IR are strongly affected by the errors in the channel estimation, while the performance gap with the proposed schemes is strongly accentuated. This phenomenon is caused by the fact that CEO-IR aims to minimize the MUI over desired symbols with unitary energy, hence leading to received points that are more prone to noise and CSI errors. ${ }^{6}$ On the other hand, it is important to notice how the performance trend of the proposed CEO-CIO scheme follows the one of the system where perfect CSI is available at the transmitter. This is due to the interference energy exploitation in the CIO scheme, which allows a certain robustness against noise in the channel estimation. In addition, we can see that the robust relaxation of CEO-CIO, identified as CEO-CIO $\mathrm{R}$ in the legend, is able to increase the performances achieved by its non-robust counterpart, due to the proposed errorbased optimization region, which allows to partially reduce the deteriorating effects of imperfect CSI at the transmitter side. Finally, it can be noticed how the CVX-CIO is inherently more robust to imperfect estimations of the CSI when compared to the approach based on the CEM-solver. This behavior is caused by the fact that CVX-CIO received signals tend be more aligned to the corresponding desired constellation points, hence allowing a innate robustness against noisy channels.

Fig. 8 studies the behavior of the proposed robust relaxation of CEO-CIO with increasing values of the error bound $\delta^{2}$ and fixed $S N R=10 \mathrm{~dB}$. As we can see, all the techniques achieve lower SER performances as the error bound $\delta^{2}$ grows. However, it is interesting to notice how the CSI errorbased region relaxation allows CEO-CIO $\mathrm{R}$ to outperform the non-robust approach over all the spectrum of $\delta^{2}$ values.

\footnotetext{
${ }^{6}$ More specifically, CEP-IR leads to received symbols that are more susceptible to the imperfect CSI shift $\bar{z}_{m}$ because of their shorter distance from the decision threshold, when compared to the proposed schemes.
} 


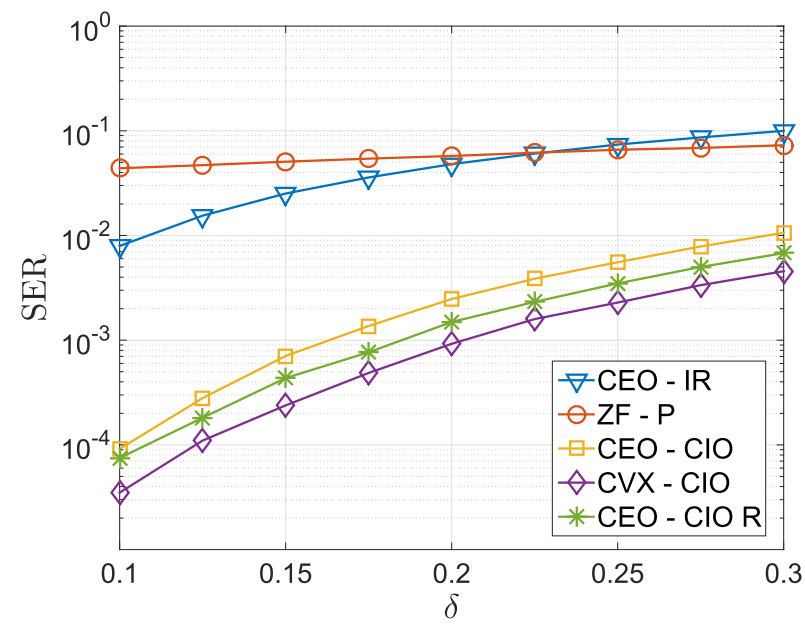

Fig. 8. Symbol error rate for 8-PSK modulation when $M=12, N=64$ and $S N R=10 d B$ with imperfect CSI.

Moreover, we can see that the performance gap between the robust and non-robust version of CEO-CIO becomes more significant as the uncertainty over the CSI grows, while the gap between CEO-CIO R and CVX-CIO reduces as $\delta^{2}$ grows. This is explained by the fact that when the error bound $\delta^{2}$ is very low, the deriving relaxation is less appreciable, hence leading CEO-CIO $\mathrm{R}$ to achieve similar performances to its non-robust counterpart. On the other hand, for higher values of $\delta^{2}$, the CEO-CIO $\mathrm{R}$ is characterized by a more noticeable relaxation which leads to higher benefits, when compared to CEO-CIO.

In order to better represent the trade-off between complexity and performances offered by the proposed scheme CEO-CIO, the achievable Symbol Error Rate (SER) is shown in Fig. 9 as a function of the per-frame flops count when considering an $S N R=0 d B$ and perfect CSI at the transmitter. More specifically, we gradually increase the size of the set of random states $K$, which directly affects the flops count per-frame shown in Table I. ${ }^{7}$ It is extremely important to highlight that, while the proposed scheme is required to evaluate the precoded signal at a symbol-rate, such need is shared by all the other CEP precoding schemes from the literature, for both single [15] and multiuser scenarios [16]-[19]. As a consequence, we can see that for similar complexities, the proposed CEO scheme is able to strongly outperform its interference reduction counterpart, hence showing a very positive and interesting trade-off between complexity and performances.

\section{A. Constellation Energy}

In our simulations we assume the desired symbols to have unitary energy constellation, i.e., $d_{m}=d=1, \forall m \in$ $\{1, \ldots, M\}$. While this assumption is not uncommon in CEP literature [15]-[17], [19], the constellation energy can be increased to improve CEP-IR performances. This represents one of the key drawbacks of the CEP-IR approach, as its

\footnotetext{
${ }^{7}$ When computing the flops per-frame, for the sake of simplicity, we consider the frame length to be equal to the coherence time for download transmission $T_{D L}=70$, in line with the LTE standard [33].
}

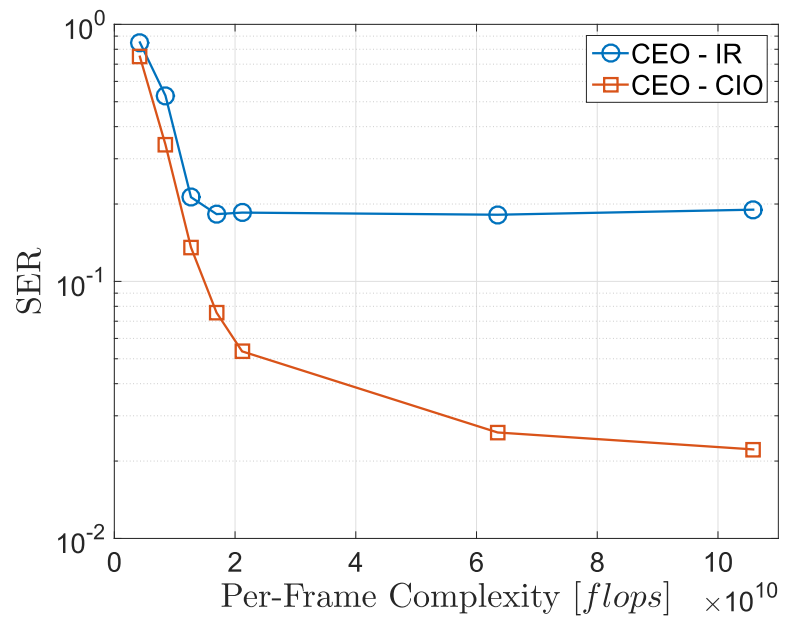

Fig. 9. Symbol Error Rate for 8-PSK modulation when $M=6$ and $N=32$ and $S N R=0 d B$ as a function of flops per-frame.

performances are strongly dependent on the constellation energy $E=d^{2}$. In fact, since the expected value of the MUI is a function of both topology (i.e., number of antennas at the BS and number of users) and modulation used in transmission [16], it is not possible to know a priori the optimal constellation amplitude $d^{*}$. More specifically, the identification of the optimal energy would require to dynamically estimate the SER at the transmitter side as a function of the constellation energy $E$, hence increasing the computational complexity of the system. Otherwise, the search for a suboptimal constellation energy for CEO-IR could be performed at the transmitter side via an additional topology-dependent optimization problem [16]. The optimization problem that identifies the optimal constellation amplitude $d^{*}$ is defined as follows [16]

$$
\begin{gathered}
\text { maximize } d \\
\text { subject to } E\left\{\sum_{m=1}^{M}\left|\left(\sum_{n=1}^{N} \frac{h_{m, n}}{\sqrt{N}} e^{j \theta_{n}}-d_{m} e^{j \phi_{m}}\right)\right|^{2}\right\} \leq \gamma \\
d_{m}=d, \quad \forall m \in\{1, \ldots, M\},
\end{gathered}
$$

where $\gamma \geq 0 \in \mathbb{R}^{+}$is a chosen threshold parameter to the MUI energy. The optimization problem aims to identify the maximum constellation energy that preserves the expected MUI energy within a desired threshold.

It is important to stress that for classic CEO-IR, the choice of the constellation energy is critical. These considerations are visually presented in Fig. 10 and Fig. 11, for the $M=6$, $N=32$ and $M=12, N=64$ scenario respectively. Both figures consider the perfect-CSI case, while similar results can be seen for the imperfect-CSI case. In fact, the aforementioned figures show that the performances of CEO-IR worsen as we incautiously increase the constellation energy $E$, with this effect being particularly visible for higher modulation orders such as 8-PSK. This is due to the fact that the MUI-based metric used for CEO-IR aims to minimize the energy of the interference signal (i.e., the distance between the received symbol and the corresponding desired constellation point), but fails to have any control over its 


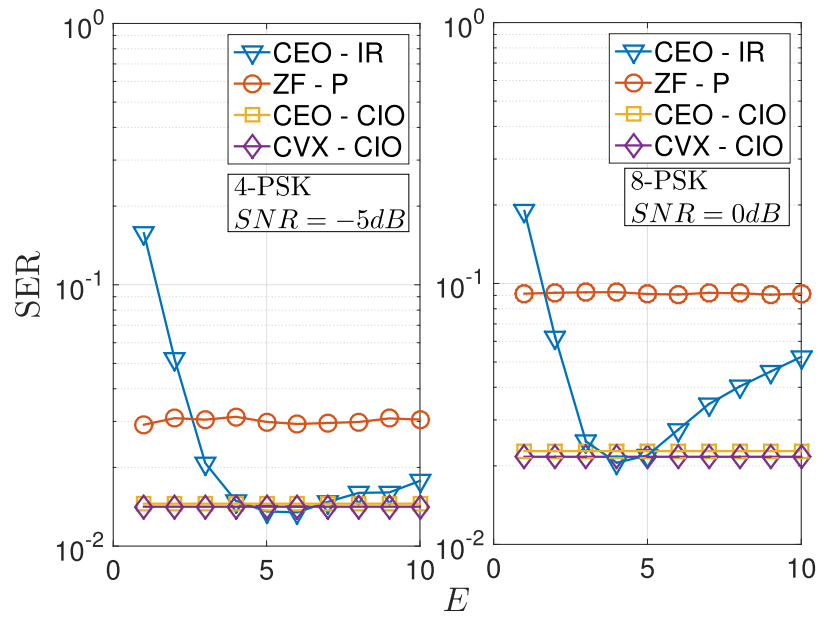

Fig. 10. Symbol Error Rate as a function of the constellation energy $E=d_{m}^{2}=d^{2}, \forall m \in\{1, \ldots, M\}$ when $M=6$ and $N=32$.

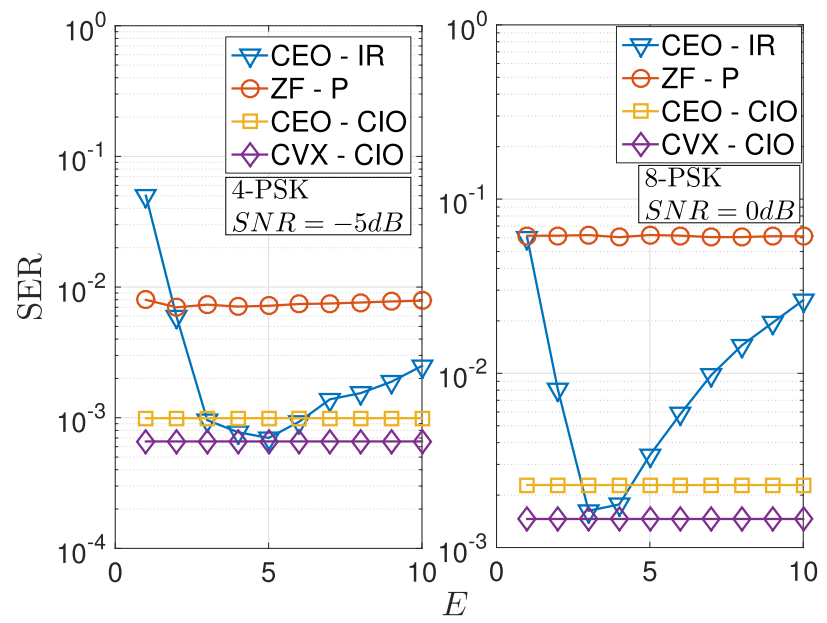

Fig. 11. Symbol Error Rate as a function of the constellation energy $E=d_{m}^{2}=d^{2}, \forall m \in\{1, \ldots, M\}$ when $M=12$ and $N=64$.

phase $\mathfrak{U}\left\{t_{m}\right\}{ }^{8}$ Moreover, we can see that the optimal $d^{*}$ changes when considering different scenarios and different modulations, supporting how it is not possible to identify $d^{*}$ before transmitting. On the other hand, the performances of the proposed techniques are not affected by the desired symbol energy, as they aim to maximize the constructive effects of interference over the received signal. Therefore, a critical benefit of the proposed scheme is that the additional optimization of $E$ can be avoided, along with the significant associated computational costs. In fact, as shown in Fig. 10 and Fig. 11 the proposed techniques are able to outperform the classical CEO-IR for most of the energy spectrum. This is supported by the fact that the performances of the proposed metric are independent from the desired symbol energy as they allow a constrained portion of the interference at the user side. In other words, the proposed metric adaptively increases the received constellation in function of

\footnotetext{
${ }^{8}$ More specifically, CEO-IR metric is not affected by the phase of the received signal, which is particularly important for PSK modulated signals, as information is carried through the phases of the received signals, while their amplitude identifies their robustness against noise.
}

the current CSI, without the need to additionally identify the optimal transmitted constellation energy, hence showing a very positive complexity-performance trade-off. Additionally, we can notice that the performance gap between CVX-CIO and CEO-CIO is larger for Fig. 11. This phenomenon is caused by aforementioned different impact of the second-step normalization of CVX-CIO, which causes the performance gap to be more significant and beneficial for CVX-CIO when considering systems with larger arrays at the BS.

\section{CONCLUSIONS}

This paper proposes a CEP scheme where multi-user interference is effectively exploited to increase the performances of systems with constant envelope constraints at the base station. The proposed techniques show that a relaxation of the optimization region in function of the constructive interference can be beneficial to achieve reliable communications. The computational burdens of the proposed techniques has been analyzed in terms of flops, and compared with the approaches from the literature, showing negligible differences. In addition, a precoding approach robust to bounded CSI errors that does not require to increase the transmitted power has been analytically derived for scenarios that involve imperfect CSI. Finally, performances have been shown in terms of symbol error rate for different modulation orders, proving the benefits introduced by the proposed scheme when compared to classical CEP approaches.

\section{REFERENCES}

[1] T. L. Marzetta, "Noncooperative cellular wireless with unlimited numbers of base station antennas," IEEE Trans. Wireless Commun., vol. 9, no. 11 , pp. 3590-3600, Nov. 2010.

[2] E. G. Larsson, O. Edfors, F. Tufvesson, and T. L. Marzetta, "Massive MIMO for next generation wireless systems," IEEE Commun. Mag., vol. 52, no. 2, pp. 186-195, Feb. 2014.

[3] J. Hoydis, S. ten Brink, and M. Debbah, "Massive MIMO in the UL/DL of cellular networks: How many antennas do we need?' IEEE J. Sel. Areas Commun., vol. 31, no. 2, pp. 160-171, Feb. 2013.

[4] A. Kammoun, A. Müller, E. Björnson, and M. Debbah, "Linear precoding based on polynomial expansion: Large-Scale multi-cell MIMO systems," IEEE J. Sel. Topics Signal Process., vol. 8, no. 5, pp. 861-875, Oct. 2014.

[5] F. Rusek et al., "Scaling up MIMO: Opportunities and challenges with very large arrays," IEEE Signal Process. Mag., vol. 30, no. 1, pp. 40-60, Jan. 2013.

[6] C. Masouros and M. Matthaiou, "Space-constrained massive MIMO: Hitting the wall of favorable propagation," IEEE Commun. Lett., vol. 19, no. 5, pp. 771-774, May 2015.

[7] S. Biswas, C. Masouros, and T. Ratnarajah, "Performance analysis of large multiuser MIMO systems with space-constrained 2-D antenna arrays," IEEE Trans. Wireless Commun., vol. 15, no. 5, pp. 3492-3505, May 2016.

[8] C. Masouros, M. Sellathurai, and T. Ratnarajah, "Large-scale MIMO transmitters in fixed physical spaces: The effect of transmit correlation and mutual coupling," IEEE Trans. Commun., vol. 61, no. 7, pp. 2794-2804, Jul. 2013.

[9] A. Garcia-Rodriguez and C. Masouros, "Exploiting the increasing correlation of space constrained massive MIMO for CSI relaxation," IEEE Trans. Commun., vol. 64, no. 4, pp. 1572-1587, Apr. 2016.

[10] M. Joham, W. Utschick, and J. A. Nossek, "Linear transmit processing in MIMO communications systems," IEEE Trans. Signal Process., vol. 53 , no. 8, pp. 2700-2712, Aug. 2005.

[11] C. B. Peel, B. M. Hochwald, and A. L. Swindlehurst, "A vector-perturbation technique for near-capacity multiantenna multiuser communication-Part I: Channel inversion and regularization," IEEE Trans. Commun., vol. 53, no. 1, pp. 195-202, Jan. 2005. 
[12] V. Mancuso and S. Alouf, "Reducing costs and pollution in cellular networks," IEEE Commun. Mag., vol. 49, no. 8, pp. 63-71, Aug. 2011.

[13] S. H. Han and J. H. Lee, "An overview of peak-to-average power ratio reduction techniques for multicarrier transmission," IEEE Wireless Commun., vol. 12, no. 2, pp. 56-65, Apr. 2005.

[14] C. Studer and E. G. Larsson, "PAR-aware large-scale multi-user MIMOOFDM downlink," IEEE J. Sel. Areas Commun., vol. 31, no. 2, pp. 303-313, Feb. 2013.

[15] S. K. Mohammed and E. G. Larsson, "Single-user beamforming in largescale MISO systems with per-antenna constant-envelope constraints: The doughnut channel," IEEE Trans. Wireless Commun., vol. 11, no. 11, pp. 3992-4005, Nov. 2012.

[16] S. K. Mohammed and E. G. Larsson, "Per-antenna constant envelope precoding for large multi-user MIMO systems," IEEE Trans. Commun., vol. 61, no. 3, pp. 1059-1071, Mar. 2012.

[17] S. K. Mohammed and E. G. Larsson, "Constant-envelope multi-user precoding for frequency-selective massive MIMO systems," IEEE Wireless Commun. Lett., vol. 2, no. 5, pp. 547-550, Oct. 2013.

[18] S. Mukherjee and S. K. Mohammed, "Constant-envelope precoding with time-variation constraint on the transmitted phase angles," IEEE Wireless Commun. Lett., vol. 4, no. 2, pp. 221-224, Apr. 2015.

[19] J. C. Chen, C. K. Wen, and K. K. Wong, "Improved constant envelope multiuser precoding for massive MIMO systems," IEEE Commun. Lett., vol. 18 , no. 8, pp. 1311-1314, Aug. 2014.

[20] V. Nguyen and J. Evans, "Multiuser transmit beamforming via regularized channel inversion: A large system analysis," in Proc. IEEE GLOBECOM, Nov./Dec. 2008, pp. 1-4.

[21] B. M. Hochwald, C. B. Peel, and A. L. Swindlehurst, "A vector-perturbation technique for near-capacity multiantenna multiuser communication-Part II: Perturbation," IEEE Trans. Commun., vol. 53, no. 3, pp. 537-544, Mar. 2005.

[22] C. Masouros, T. Ratnarajah, M. Sellathurai, C. B. Papadias, and A. K. Shukla, "Known interference in the cellular downlink: A performance limiting factor or a source of green signal power?" IEEE Commun. Mag., vol. 51, no. 10, pp. 162-171, Oct. 2013.

[23] C. Masouros and E. Alsusa, "Dynamic linear precoding for the exploitation of known interference in MIMO broadcast systems," IEEE Trans. Wireless Commun., vol. 8, no. 3, pp. 1396-1404, Mar. 2009.

[24] C. Masouros and E. Alsusa, "Soft linear precoding for the downlink of DS/CDMA communication systems," IEEE Trans. Veh. Technol., vol. 59, no. 1, pp. 203-215, Jan. 2010.

[25] C. Masouros, "Correlation rotation linear precoding for MIMO broadcast communications," IEEE Trans. Signal Process., vol. 59, no. 1, pp. 252-262, Jan. 2011

[26] C. Masouros and G. Zheng, "Exploiting known interference as green signal power for downlink beamforming optimization," IEEE Trans. Signal Process., vol. 63, no. 14, pp. 3628-3640, Jul. 2015.

[27] M. Alodeh, S. Chatzinotas, and B. Ottersten, "Constructive multiuser interference in symbol level precoding for the MISO downlink channel," IEEE Trans. Signal Process., vol. 63, no. 9, pp. 2239-2252, May 2015.

[28] M. Alodeh, S. Chatzinotas, and B. Ottersten, "Energy-efficient symbollevel precoding in multiuser MISO based on relaxed detection region," IEEE Trans. Wireless Commun., vol. 15, no. 5, pp. 3755-3767, May 2016.

[29] A. Li and C. Masouros, "Exploiting constructive mutual coupling in P2P MIMO by analog-digital phase alignment," IEEE Trans. Wireless Commun., to be published.

[30] K. L. Law, C. Masouros, K. K. Wong, and G. Zheng, "Constructive interference exploitation for outage-probability constrained downlink precoding optimization," IEEE Trans. Signal Process., to be published.

[31] A. Kalantari, M. Soltanalian, S. Maleki, S. Chatzinotas, and B. Ottersten. (2016). "Directional modulation via symbol-level precoding: A way to enhance security." [Online]. Available: https://arxiv.org/abs/1606.04488

[32] M. P. Daly and J. T. Bernhard, "Directional modulation technique for phased arrays," IEEE Trans. Antennas Propag., vol. 57, no. 9, pp. 2633-2640, Sep. 2009.

[33] Evolved Universal Terrestrial Radio Access (E-UTRA); Physical Channels Modulation, document 3GPP TS 36.211, V8.2.0 Release 8, 2008.

[34] D. Kwon, H. S. Kang, and D. K. Kim, "Robust interference exploitationbased precoding scheme with quantized CSIT," IEEE Commun. Lett., vol. 20, no. 4, pp. 780-783, Apr. 2016.
[35] M. Alodeh, S. Chatzinotas, and B. Ottersten. (2016). "Symbol-level multiuser MISO precoding for multi-level adaptive modulation: A multicast view." [Online]. Available: https://arxiv.org/abs/1601.02788

[36] H. Yang and T. L. Marzetta, "Performance of conjugate and zeroforcing beamforming in large-scale antenna systems," IEEE J. Sel. Areas Commun., vol. 31, no. 2, pp. 172-179, Feb. 2013.

[37] P.-T. de Boer, D. P. Kroese, S. Mannor, and R. Y. Rubinstein, "A tutorial on the cross-entropy method," Ann. Oper. Res., vol. 134, no. 1, pp. 19-67, 2005.

[38] R. Rubinstein and D. Kroese, The Cross-Entropy Method: A Unified Approach to Combinatorial Optimization, Monte-Carlo Simulation and Machine Learning (Information Science and Statistics). New York, NY, USA: Springer, 2011.

[39] S. Boyd and L. Vandenberghe, Convex Optimization. Cambridge, U.K.: Cambridge Univ. Press, 2010.

[40] T. L. Marzetta, "How much training is required for multiuser MIMO?" in Proc. Asilomar Conf. Signals, Syst. Comput. (ACSSC), 2006, pp. 359-363.

[41] N. Vucic and H. Boche, "Robust QoS-constrained optimization of downlink multiuser MISO systems," IEEE Trans. Signal Process., vol. 57, no. 2, pp. 714-725, Feb. 2009.

[42] B. K. Chalise, S. Shahbazpanahi, A. Czylwik, and A. B. Gershman, "Robust downlink beamforming based on outage probability specifications," IEEE Trans. Wireless Commun., vol. 6, no. 10, pp. 3498-3503, Oct. 2007.

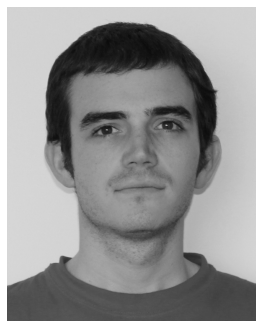

Pierluigi Vito Amadori (S'14) received the M.Sc. degree (Hons.) in telecommunications engineering from the University of Rome La Sapienza, Rome, Italy, in 2013. He is currently pursuing the $\mathrm{Ph} . \mathrm{D}$. degree with the Department of Electrical and Electronic Engineering, University College London, London, U.K. From 2012 to 2013, he held a JPL Visiting Student Researchers Program position with the Jet Propulsion Laboratory Pasadena, Pasadena, CA, USA. His main research interests include wireless communications with a focus on large antenna array systems and energy efficient communications.

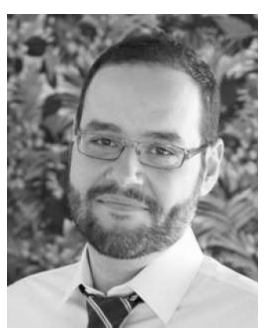

Christos Masouros (M'06-SM'14) received the Diploma degree in electrical and computer engineering from the University of Patras, Greece, in 2004, and the M.Sc. and Ph.D. degrees in electrical and electronic engineering from the University of Manchester, U.K., in 2006 and 2009, respectively. $\mathrm{He}$ held a Research Associate position with the University of Manchester, U.K., and a Research Fellow position with Queen's University Belfast, U.K. He is currently a Senior Lecturer with the Department of Electrical and Electronic Engineering, University College London. He held a Royal Academy of Engineering Research Fellowship from 2011 to 2016.

His research interests lie in the field of wireless communications and signal processing with particular focus on Green communications, cognitive radio, and interference mitigation techniques for MIMO. He was a recipient of the Best Paper Award in the IEEE GLOBECOM Conference in 2015. He is the Principal Investigator of the EPSRC Project EP/M014150/1 on large scale antenna systems. He is an Associate Editor of the IEEE COMMUNICATIONS LETTERS and has been a Guest Editor of the IEEE JOURNAL ON SELECTED TOPICS IN Signal Processing Special Issue on Exploiting Interference toward Energy Efficient and Secure Wireless Communications. He has been recognized as an Exemplary Reviewer of the IEEE TRANSACTIONS ON COMMUNICATIONS. 